\title{
DETERMINANTES SOCIALES DE LA SALUD Y RIESGO DE DIABETES TIPO 2 EN ADULTOS DE POBLACIONES ORIGINARIAS, APROXIMACIONES DESDE LA TEORÍA SOCIAL
}

\author{
SOCIAL DETERMINANTS OF HEALTH AND RISK OF TYPE 2 DIABETES IN \\ ADULTS FROM INDIGENOUS POPULATIONS, APPROACHES FROM SOCIAL \\ THEORY
}

\author{
Félix Gerardo BUICHIA SOMBRA ${ }^{1}$ \\ Guadalupe Adriana MIRANDA COTA ${ }^{2}$
}

\begin{tabular}{|lll|}
\hline Recibido & $:$ & 30.11 .2020 \\
Aceptado & $:$ & 08.01 .2021 \\
Publicado & $:$ & 12.02 .2021 \\
\hline
\end{tabular}

\begin{abstract}
RESUMEN: La Diabetes Tipo 2 (DT2) es una enfermedad multicausal que requiere de estudios desde diversas perspectivas: biológicas, psicológicas, sociales y culturales, resaltar que la salud no solo es un proceso de orden bionatural, sino que, es una cuestión social compleja, que además ha sido poco abordada. El objetivo es exponer los Determinantes Sociales de la Salud (DSS) y riesgo de diabetes en población originaria, a partir de la teoría social, específicamente de la corriente epistemológica del configuracionismo: Estructura, Subjetividad y Acción, desde la propuesta Giddens y de la Garza, a partir de estos conceptos, se presenta un esquema conceptual sobre los DSS en el riesgo de DT2 en población originaria. Se concluye que es necesario realizar estudios sociales de la experiencia de vida tratando de encontrar las conexiones entre las estructuras y cómo estas se posicionan sobre el cuidado de la salud y prevención de enfermedades, a ello se suman la multiplicidad de significados que cada persona le da a la salud, su cuidado y el afrontamiento de la enfermedad.
\end{abstract}

Palabras clave: Diabetes Tipo 2, Teoría Social, riesgo, población indígena.

\begin{abstract}
Type 2 Diabetes (T2D) is a multiclausal disease that requires studies from different perspectives: biological, psychological, social and cultural, highlighting that health is not only a bio natural process, but that it is a complex social issue, which has also been little addressed. The objective is to expose the Social Determinants of Health ( $\mathrm{SDOH})$ and risk of diabetes in the native population, based on social theory, specifically the epistemological current of configurationism: Structure, Subjectivity and Action, from the proposal Giddens and de la Garza Based on these concepts, a conceptual scheme is presented on the DSS in the risk of T2D in the native population. It is concluded that it is necessary to carry out social studies of the life experience trying to find the connections between the structures and how they are positioned on health care and disease prevention, to this is added the multiplicity of meanings that each person gives to health, its care and coping with illness.
\end{abstract}

Keywords: Type 2 Diabetes, Social Theory, risk, indigenous population.

\footnotetext{
${ }^{1}$ Doctorado en Estudios Sociales. Universidad Autónoma Indígena de México. Estado de Sinaloa-México Email: buichiasombraf@gmail.com ORCID: https://orcid.org/0000-0003-1256-1828

${ }^{2}$ Licenciada en Enfermería. Universidad Autónoma Indígena de México. Estado de Sinaloa-México. Email: mlupita09@gmail.com.ORCID: https://orcid.org/0000-0002-4322-2215
} 


\section{INTRODUCCIÓN}

La Diabetes Tipo 2 (DT2) es una enfermedad de etiología múltiple y considerada actualmente una emergencia de salud pública debido a su prevalencia creciente y altas tasas de mortalidad, generando en los individuos, familias, a la sociedad y sistemas de salud, costos económicos y sociales altos para su prevención, control y tratamiento. A pesar de que las instituciones de salud han realizado esfuerzos para mejorar el panorama de la diabetes, este problema de salud sigue al alza y con proyecciones poco favorables, lo que indica que, su etiología requiere de un abordaje integral y estudios que expliquen desde diversas perspectivas, como las ciencias sociales, que permitan dimensionar el impacto de los factores socioculturales y los Determinantes Sociales de la Salud (DSS) para el riesgo de DT2, sobre todo en grupos minoritarios, entre los que destacan las poblaciones originarias (Federación Internacional de la Diabetes [IDF], 2019; Organización Mundial de la Salud [OMS], 2016).

La OMS (2018) señaló a la diabetes como causa importante de ceguera, ataques cardiacos, insuficiencia renal, apoplejía y amputación de extremidades inferiores. Aunado al deterioro progresivo que produce la enfermedad en las personas, sus familiares y la comunidad. Por lo anterior, evaluar el riesgo actual y futuro de la diabetes es elemental para dimensionar el impacto de los programas de atención específicos, organizar apropiadamente el uso de recursos en salud, promover políticas públicas inclusivas de promoción de salud adaptadas a cada grupo poblacional e impulsar estrategias socioculturales para prevenir la enfermedad.

En este sentido, estudiar la posición social de una persona sobre y desde las estructuras sociales permite el análisis sobre el origen e impacto en circunstancias que le afectan o le benefician, de la posición social podrían surgir las inequidades en salud, estas se definen como la ausencia de disparidades entre grupos, determinados por procesos sociales, económicos, demográficos o geográficos, que ponen en desventaja a unos frente a otros, particularmente respecto a la salud, que se diferencian entre ellos por un distinto nivel de poder, bienestar o prestigio social (Linares y López, 2003). Dichas diferencias son potencialmente modificables en uno o más aspectos: salud, educación, nutrición y condiciones de vida. La equidad es un concepto cercano a la noción de justicia distributiva como respuesta a necesidades y demandas de las poblaciones (Cardona, Acosta y Bertone, 2013; Meljem, 2016). 


\section{Journal of the Academy $\mid 229$ |}

La OMS (2009) ha descrito como las inequidades afectan a la salud de las sociedades y ha puesto en marcha políticas públicas y programas para disminuir las inequidades que afectan los resultados de la salud-enfermedad, de tal manera que, los DSS podrían dar luz respecto a la explicación de cómo las circunstancias en que las personas nacen, crecen, viven, trabajan y envejecen, incluido el sistema de salud. Esas circunstancias son el resultado de la distribución del dinero, el poder y los recursos a nivel mundial, nacional y local, que depende a su vez de las políticas adoptadas. A través de los DSS se podrían explican las inequidades sanitarias, es decir, las diferencias injustas y evitables observadas en y entre los países ante el panorama epidemiológico particular.

Estas inequidades se ven en mayor proporción en población de estratos sociales bajos, entre los que se encuentran las poblaciones originarias, quienes, a pesar de su heterogeneidad, son vulnerables a desarrollar DT2. Lo que podría deberse a la influencia de componentes de inequidad social en salud, en educación y económicos, sumado a los cambios ocurridos por la urbanización y aculturación al interior y exterior de las comunidades que impactan en el estilo de vida como la dieta poco saludable y la disminución de la actividad física (Egeland et al., 2011; Esparza et al., 2015). Además, es evidente que los datos anteriores no incluyen categorías que permitan identificar la subjetividad del sujeto, es decir aquellos significados, creencias, valores y costumbres que configuran el hábitat de la población originaria y que podrían describir a profundidad las acciones que esta población realiza para el cuidado de su salud y afrontamiento de la enfermedad.

En lo que corresponde a la población Yoreme-Mayo del norte de Sinaloa, actualmente no se disponen de datos sobre el riesgo de DT2, por lo que se desconoce la magnitud del problema y el estado de afectación de la población. Asimismo, no se cuenta con información respecto a cómo los DSS se relacionan con el riesgo de DT2, que ayuden a dilucidar y conocer el panorama de riesgo en esta población. Por lo que resulta interesante analizar desde la teoría configuracionista: estructura, subjetividad y acción, un esquema conceptual que nos permita identificar las categorías que podrán ayudarnos a la comprensión del horizonte de riesgo para DT2 en población originaria Yoreme-Mayo. Pero antes de abordar lo anterior, es importante identificar el panorama general de la DT2, el cual se describe a continuación. 


\section{DESARROLLO}

\section{La Diabetes Mellitus}

De acuerdo con la Asociación Latinoamericana de Diabetes (ALAD, 2019) el término diabetes mellitus (DM) se refiere a un desorden metabólico de etiología múltiple que se caracteriza por hiperglucemia crónica con alteración en el metabolismo de los carbohidratos, grasas y proteínas y que resulta de defectos en la secreción y/o en la acción de la insulina.

\section{Clasificación y definición de la Diabetes Mellitus}

La ADA (2020) categoriza a la DM en las siguientes categorías generales: (1) Diabetes tipo 1, cuya fisiopatología se explica debido a la destrucción de células autoinmunes, que generalmente conduce a una enfermedad absoluta deficiencia de insulina, (2) Diabetes tipo 2 (DT2), que ocurre debido a una pérdida progresiva de la secreción adecuada de insulina de células B que conlleva a la resistencia a la insulina, (3) DM gestacional, diabetes diagnosticada en el segundo o tercer trimestre del embarazo sin antecedentes de diagnóstico de diabetes antes de la gestación y (4) Tipos específicos de diabetes debido a otras causas, síndromes de diabetes monogénica, enfermedades del páncreas exocrino (fibrosis quística y la pancreatitis) y el medicamento o diabetes inducida por sustancias químicas (como con el uso de glucocorticoides, en el tratamiento de VIH/SIDA, o después de un trasplante de órgano).

De la clasificación anterior, resalta que la DT2 representa del 90\% al 95\% de los casos de DM en el mundo, lo que indica una fuerte carga para el grupo de adultos de 20 años y más, que además viven en países de ingresos económicos medios y bajos, por ende, la convierte en un problema de salud con dimensiones sociales importantes (OMS, 2016).

\section{Factores Biológicos Asociados a Diabetes Tipo 2}

Diversas organizaciones internacionales han publicado factores que podrían estar asociados a DT2, entre ellos: tener sobrepeso u obesidad, tener más de 45 años de edad, historia familiar de diabetes, ser afroamericano, nativo de Alaska, indígena estadounidense, estadounidense de origen asiático, hispana/latina, nativa de Hawái, o de las Islas del Pacífico. Además de datos 


\section{Journal of the Academy | 231 |}

clínicos, como hipertensión arterial sistémica, nivel bajo de colesterol HDL o un nivel alto de triglicéridos, antecedentes de diabetes gestacional o tener un hijo que pesó más de 4000 gramos al nacer, antecedentes de enfermedades del corazón o accidentes cardiovasculares o acantosis nigricans. Factores ambientales, como actividad física leve o nula y factores emocionales, como la depresión (ADA, 2020; 2019; IDF, 2019; Instituto Nacional de Diabetes y Enfermedades Digestivas y Renales, 2020). Los factores anteriores no involucran factores sociales, sin embargo, estos últimos podrían desencadenar o ser mediadores sobre algunos de los factores propuestos por los organismos antes citados.

\section{Complicaciones de la Diabetes Tipo 2}

Las personas con diabetes que no tienen un control adecuado de la enfermedad tienen un alto riesgo de presentar un conjunto de problemas de salud potencialmente mortales (IDF, 2019). Las complicaciones pueden ser desde agudas y/o crónicas. Estas últimas surgen con el transcurso de los años y es común que exista daño antes de que se presenten signos y síntomas. Estas se dividen en complicaciones macrovasculares y microvasculares.

\section{Panorama Epidemiológico de la DT2}

La IDF (2019) estimó que en el mundo existían 463 millones de personas que viven con diabetes, de éstas, aproximadamente el 79\% vive en países de ingresos bajos y medios, entre ellos México. Las proyecciones para el año 2045 apuntan a que habrá aproximadamente 700 millones de adultos con esta enfermedad. En el año 2019, México se posicionó como el sexto país con más personas de 20 a 79 años con diabetes antecedido por China, India, Estados Unidos, Pakistán y Brasil.

En el mundo se calcula que 4.2 millones de adultos de entre 20 y 79 años murieron como resultado de la diabetes y sus complicaciones en 2019. Esto equivale a una muerte cada ocho segundos. Se calcula que la diabetes se asocia con el $11.3 \%$ de los fallecimientos a nivel mundial por todas las causas posibles entre las personas de este grupo de edad. Casi la mitad (46.2\%) de las muertes asociadas con la diabetes entre el grupo de edad de entre 20 y 79 años se producen en personas menores de 60 años, es decir, el grupo de edad económicamente productiva (IDF, 2019). 


\section{Journal of the Academy $|232|$}

La carga de la DT2 recae en grupos socialmente desfavorecidos como los pueblos originarios, quienes históricamente han sido oprimidos por grupos de poder, causando con ellos injusticia, inequidad, pobreza, lo cual los vulnera frente a circunstancias relacionadas con el cuidado y resultados de la salud. Los grupos representan el sector minoritario más grande al estimarse que 370 millones de personas habitan en más de 90 países, entre ellos México. En el país se tiene registro de 68 pueblos originarios que representan al $10.1 \%$ de la población, de los pueblos originarios registrados en México, la población Yoreme-Mayo es la más representativa del norte de Sinaloa, la cual se encuentra dispersa principalmente en los municipios de Sinaloa de Leyva, Guasave, Ahome, el Fuerte y Choix (Banco Mundial, 2018; Instituto Nacional de los Pueblos Indígenas [INPI], 2019).

En México, en lo que corresponde la magnitud de la DT2, la prevalencia de DT2 previa en población general ha tenido un aumento del año 2000 a 2018, al pasar de 5.8\% a $10.3 \%$. En esta última, la mayor prevalencia se observó en personas mayores de 50 años, siendo superior en mujeres tanto en zonas rurales como urbanas. En Sinaloa la situación no es diferente, en 2012 se estimó que el 8.2\% de la población padecía DT2, a pesar de que se encuentra por debajo de la media nacional, la prevalencia ha tenido un leve aumento de 2006 a 2012, esta situación supone un alto impacto económico y social para la región (ENSANUT, 2018; 2016; 2012).

En cuanto a la DT2 en población originaria, se ha documentado que en México se tienen altas tasas de esta enfermedad y se han identificado diversos componentes, como debido los genéticos, factores de inequidad social (despojo, exclusión, discriminación), un bajo nivel socioeconómico, menos años de escolaridad y un limitado acceso a los servicios de salud, además de cambios en el estilo de vida (Basilio, Kwan y Towers, 2016; Caballero, 2018; Egeland y Young, 2011; Juárez-Ramírez et al., 2019 Leyva-Flores, Infante-Xibille, Gutiérrez y Quintino-Perez, 2013).

Los datos disponibles sobre prevalencia de DT2 en población indígena de México son de estudios realizados entre 2017 y 2019 en Mixtecos de Baja California, Pimas y Yaquis de Sonora, Mayas de Yucatán y Tojolabal de Chiapas, cuyas prevalencias oscilan entre el 4 y 26\%. En estas mismas poblaciones se ha encontrado que la historia familiar de DT2, tener 


\section{Journal of the Academy $|233|$}

sobrepeso y obesidad, menos años de escolaridad, mayor edad, hipertensión arterial y ser mujer se asocian como factores de riesgo para DT2 (Castro et al., 2018; Esparza et al., 2015; Jiménez et al., 2019; Loria et al, 2018; Pacheco, et al., 2018).

La interacción entre las desigualdades y la salud es compleja: mejores resultados económicos y educativos para los hogares mejoran la salud, el bajo nivel socioeconómico conduce a enfermedades crónicas y enfermedades no transmisibles, entre las que destaca la DT2. Las enfermedades no transmisibles, específicamente la DT2 representa la mayoría de las causas de muerte prematura y discapacidad en todo el mundo, por lo que es alarmante que una fuerte evidencia científica sugiera un aumento en la agrupación de condiciones no transmisibles con bajo nivel socioeconómico en países de ingresos económicos bajos y medianos, este panorama pone de relieve la inequidad existente entre los diversos grupos sociales (Niessen et al., 2018).

Se ha evidenciado que, en México prevalecen desigualdades en acceso a servicios de salud para la población en condiciones de pobreza, esta situación impacta en la calidad de vida, al disminuir la probabilidad de recibir atención por cualquier enfermedad, incluyendo a la DT2, enfermedad que requiere de control y tratamiento de por vida (Gutiérrez et al., 2019). Otro elemento esencial es el acceso a una alimentación que permita el máximo desarrollo de la persona, en este sentido la seguridad de la alimentación en población mexicana disminuye en aquellos que tiene ingresos económicos y escolaridad considerados como bajos, situación que aumenta el riesgo de desarrollar DT2 y dificultad para el control metabólico en pacientes diagnosticados (Mundo-Rosas et al., 2019).

Por su parte Villalobos et al. (2019) reportaron que tener baja escolaridad, pertenecer al estrato económico bajo, hablar lengua indígena y tener mayor edad incrementa las posibilidades de no realizar acciones de autocuidado de la DT2. Además, pertenecer a un hogar indígena incrementa las posibilidades de que el personal de salud no realice las acciones básicas durante la consulta. El panorama anterior permite realizar un análisis respecto a las inequidades en salud, pero, además, de las experiencias y significados que las poblaciones dan a la enfermedad; a partir de la configuración de la realidad comprender la acción para la prevención de la enfermedad que surge desde y a partir de la interacción social. 


\section{Journal of the Academy | $234 \mid$}

\section{Teoría Social y Salud-Enfermedad}

Tradicionalmente, los problemas de salud se han abordado desde las ciencias naturales tratando de identificar agentes causales y su efecto en el cuerpo humano a través de la caracterización de los efectos fisiopatológicos y ofrecer un antídoto que ayude a revertir o eliminar tal efecto, esta dinámica de abordaje biomédico ha sido aceptado por la comunidad científica médica y ha permitido un avance en la compresión biológica de la enfermedad, sin embargo, no considera las estructuras, los significados, el hábitat de los sujetos y su interacción con el entorno (Engel, 1997).

Por otra parte, la reflexión sobre los problemas de salud desde una perspectiva social y cultural ha tenido un aporte a través de la práctica académica. En el caso específico de México desde los años setenta ha tenido una fuerza mayor sobre todo en el abordaje a través de la antropología y sociología (Menéndez, 2016). En Latinoamérica, diversos sociólogos de la salud han propuesto como objetivo de la medicina social el estudio de la salud-enfermedad como entidades inseparables, las cuales deben ser entendidas como dos momentos de un mismo fenómeno, en el que los sujetos desarrollan conductas (acciones) frente a la enfermedad según su realidad. En este sentido, la corriente de la medicina social enfoca el estudio de la desigualdad social y la forma que está desigualdad determina el proceso de salud-enfermedad de las poblaciones (Iriart, 2020).

Los conceptos de clase y estrato sociales son formas fundamentalmente construcciones sociales generadoras de la desigualdad. La clase social supone diferenciación entre los grupos sociales, donde es imposible la movilidad ascendente, es decir cambiar de clase social, mientras que el estrato social supone que las diferencias entre los estratos son cuantitativas, es decir se posee más o menor riqueza, donde por tanto es posible ascender o descender de la escala social (Benach y Amable, 2004).

Es aquí donde se podría abordar la situación de clase y estrato social de los grupos originarios, partiendo de la aproximación de Weber al referirse a los grupos étnicos como una construcción social cuya existencia es casi siempre problematizada. Por su parte, Bari (2002) refiere que los grupos étnicos se diferencian uno de otro a partir de un cuerpo particular de aspectos culturales, estos se movilizan, desplazan o se retraen en situaciones de relaciones de interacción, dicha 


\section{Journal of the Academy $\mid 235$ |}

interacción genera códigos culturales con significados respecto a los fenómenos que se generan en la interacción, entre ellos lo relacionado a la salud y la enfermedad.

La importancia del significado de la salud-enfermedad en los grupos sociales, se incorpora de una forma amplia ante la experiencia subjetiva y los significados de la salud-enfermedad que se articulan con las estructuras sociales y la acción. Explorar esas experiencias, permitirá comprender los resultados de la salud que los sujetos tienen para así reorganizar el abordaje de las enfermedades como la DT2 al incluir la dimensión personal y subjetiva de los sujetos, alcanzando aquí una profunda comprensión de los padecimientos.

En el mismo orden de ideas, para el abordaje de la dimensión personal y subjetiva de los sujetos sobre la enfermedad DT2, planteamos la propuesta metodológica configuracionista de De la Garza (2018), quien plantea la configuración de relaciones sociales, la configuración de estructuras, la configuración de códigos subjetivos para dar significados, que conducen a ver las relaciones entre estructuras, subjetividades y acción. Estos conceptos parten de la interpretación de Giddens, Marx y Gramsci.

\section{Estructura social}

Las estructuras condicionan las acciones para prevenir el riesgo de la enfermedad y estas son el resultado de la presencia de la enfermedad en las poblaciones. Por su parte, Bourdieu (2017) en el concepto de estructura remite al de habitus, entendido como estructuras mentales o cognitivas para manejar el mundo social, y al de campo como redes de relaciones entre posiciones objetivas; sin embargo, tanto el habitus como el campo pueden ser entendidos a partir de prácticas regulares, actualizadas permanentemente.

Para Giddens (1998) las estructuras son directamente prácticas ordenadas recurrentes, es decir, no existen en sí margen de la praxis, de tal forma que la continuidad de la estructura necesita del concepto de estructuración como reproducción de relaciones sociales negociadas a lo largo del tiempo. Trata de resolver de esta manera la dualidad entre estructura y acción, las estructuras acondicionan a la acción y a la vez son resultados o se actualizan por las prácticas, el centro es por tanto la praxis, no la conciencia ni la estructura (De la Garza, 2001, p.92). 


\section{Journal of the Academy $|236|$}

A partir de lo anterior se retoman estructuras sociales a través de los Determinantes Sociales de la Salud (DSS), específicamente sobre la DT2, partiendo del modelo propuesto por la Comisión de los Determinantes sociales de la Salud de la OMS (2009).

La determinación social de la salud es una de las tres categorías centrales de la propuesta para una epidemiología crítica, enunciada desde mediados de la década del 70 y luego desarrollada por otros autores especialmente de la corriente latinoamericana. Junto con las categorías de reproducción social y metabolismo sociedad-naturaleza han conformado el eje teórico de una propuesta de ruptura con el paradigma biomédico positivista dominante de la salud pública.

El punto de partida de esta construcción teórica se fundamenta en una relectura desde el realismo crítico de dos vertientes epistemológicas encabezadas por Kuhn y Bourdieu. Del primero, para rescatar la visión dialéctica del pensamiento científico, su naturaleza social, transformabilidad y el carácter "revolucionario" dado por la oposición de ideas innovadoras frente a los aspectos "normales", lo que abre puertas a una aproximación sociopolítica de la determinación comunitaria de la ciencia, sus modelos e instrumentos. Y el segundo, porque analiza “... la teoría como un modus operandi que orienta y organiza la práctica científica... (Y las) ...capacidades creadoras, activas, inventivas del habitus" y el lado activo del conocimiento científico como “...el capital de un sujeto trascendente de un agente en acción” (Breilh, 2013, p.13).

\section{Determinantes Sociales de la Salud}

Las Ciencias Sociales y Ciencias de la Salud se encuentran emparejadas y son producto de las condiciones históricas, Spencer definía a la sociedad como un organismo vivo, análogo a la célula. El análisis de las sociedades parte de fenómenos como la globalización que se refiere al creciente proceso de integración de las economías al mercado mundial, que es posible por el creciente desarrollo de las comunicaciones. Sin embargo, este fenómeno ha repercutido en el incremento de las desigualdades sociales, en países cuyas clases sociales gozan de una clara ventaja socioeconómica frente a grupos desfavorecidos, como históricamente han sido los grupos originarios y quienes han vivido en situación de pobreza y marginación, aumentando el riesgo de enfermar. 


\section{Journal of the Academy | $237 \mid$}

A través del análisis de los DSS se pueden explican la mayor parte de las desigualdades en salud, esto es, de las diferencias observadas en y entre los países sobre a la situación sanitaria. En respuesta a la creciente preocupación ocasionada por esas inequidades persistentes y cada vez mayores, la OMS (2008), estableció en la Comisión sobre DSS, con el objetivo de asesorar y enunciar políticas públicas para mitigarlas. En el informe final de la Comisión, publicado en 2008, se proponen tres recomendaciones generales: (1) mejorar las condiciones de vida cotidianas, (2) luchar contra la distribución desigual del poder, el dinero y los recursos y (3) medición y análisis del problema.

\section{Marco de los Determinantes sociales de la Salud, según la Organización Mundial de la} Salud.

Para poder investigar las desigualdades en salud, es necesario abordar un marco conceptual que permita comprender los mecanismos y los procesos que intervienen. El marco conceptual de los DSS propuesto combina elementos de diversos modelos y fue elaborado para satisfacer todos los criterios precitados. En este marco se presentan dos grupos de determinantes, para la mejor comprensión sobre los aspectos que determinan los procesos de salud-enfermedad de los grupos sociales: determinantes estructurales y determinantes intermediarios, que desde la teoría configuracionista se sitúan en las estructuras.

\section{Determinantes Estructurales e Intermediarios de la Salud}

Los determinantes estructurales están constituidos por aspectos relacionados con la estructura social que determinan las desigualdades en salud. Es decir, son factores que pueden colocar a los grupos sociales ante un escenario de mayor vulnerabilidad ante los procesos de salud y enfermedad, o por el contrario mayores oportunidades para recuperar o mantener la salud. Las dos situaciones anteriores dependerán de la posición social, pues grupos sociales con mayor acceso a recursos materiales, sociales, educacionales, entre otros, pueden presentar contextos menos vulnerables o con mayores posibilidades para mantener o recuperar la salud. Situación diferente, cuando la estructura social de los grupos limita el acceso a recursos que permitan mantener la salud (OMS, 2009). 


\section{Journal of the Academy $|238|$}

En este sentido, los determinantes estructurales de los DSS incluyen el contexto socioeconómico y político, así como los mecanismos que determinan la posición socioeconómica de los grupos sociales. Se consideran determinantes del contexto socioeconómico y político a todos los mecanismos sociales, económicos y políticos que generan y mantienen las posiciones socioeconómicas desiguales o la estratificación social, al asignar a los individuos diferente posición social y ésta, a su vez, determina las circunstancias materiales en que las personas viven y se desenvuelven. Destacar que su elemento central es la gobernanza (OMS, 2009).

Los mecanismos que determinan la posición socioeconómica de los grupos sociales se relacionan con la educación y el ingreso, entendiendo que el nivel educativo influye en la ocupación y ésta última en el ingreso (De la Torre-Ugarte-Guanilo y Oyola-García, 2014; OMS, 2008). La revisión de la literatura consultada permitió identificar DSS estructurales de los pueblos originarios para el riesgo de DT2 en los cuales se encuentran la escolaridad, menores ingresos, acceso a los servicios de salud, pertenecer a un grupo originario disminuye la posibilidad de recibir atención en las unidades de salud.

Determinantes Intermediarios de la Salud

Los determinantes intermediarios de la salud, se refieren a las condiciones materiales en que las personas viven y se desenvuelven, circunstancias psicosociales o socio-ambientales, factores comportamentales y al sistema de salud. En este sentido, al sistema de salud se le atribuye la responsabilidad de desempeñar una función activa para reducir las inequidades, no sólo a través del acceso equitativo a los servicios de atención (acceso, cobertura y seguimiento), sino también en la planeación e intervenciones de programas de salud pública inclusivos, con una adecuada implementación que involucre a todos los grupos sociales, especialmente a los grupos originarios (OMS, 2009).

Entre los determinantes estructurales e intermediarios se encuentra la cohesión y el capital social, puntos centrales para las políticas de salud, que se refieren al establecimiento de relaciones de cooperación entre las sociedades e instituciones de salud, principalmente. Es decir, el Estado debe asumir la responsabilidad de desarrollar un sistema más flexible que facilite espacios concretos de participación de los ciudadanos, lo que implica una redistribución del poder, que permita a la comunidad poseer un alto nivel de influencia en la toma de 


\section{Journal of the Academy | 239|}

decisiones y el desarrollo de las políticas que afectan su bienestar y calidad de vida (OMS, 2009).

Es importante resaltar que los determinantes intermediarios afectan la salud, en mayor o menor grado, de acuerdo con de la posición social, puesto que los individuos según su posición social tendrán diferentes exposiciones ante las condiciones que pueden perjudicar su salud y, por tanto, presentarán diferente vulnerabilidad, la cual determinará diferentes consecuencias ante los daños a la salud, incluyendo consecuencias económicas y sociales, así como resultados diferentes en salud-enfermedad (OMS, 2009).

En este sentido se ha documentado que los pacientes con DT2 buscan activamente atender su salud por varios medios; lo que implica que en la trayectoria de atención se mezclan modelos, recursos y medicamentos, en parte debido a las condiciones de pobreza. La evidencia puede ser usada para ajustar los programas de educación para la salud, siguiendo la propuesta de la promoción de la salud como eje estructural para favorecer e impulsar que las personas tengan mayor control de su propia salud (Juárez-Ramírez et al., 2019). En la figura 1 se muestra el marco conceptual sobre los Determinantes de Salud de la OMS (2010).

\section{Figura 1.}

Marco conceptual sobre los Determinantes Sociales de la Salud.

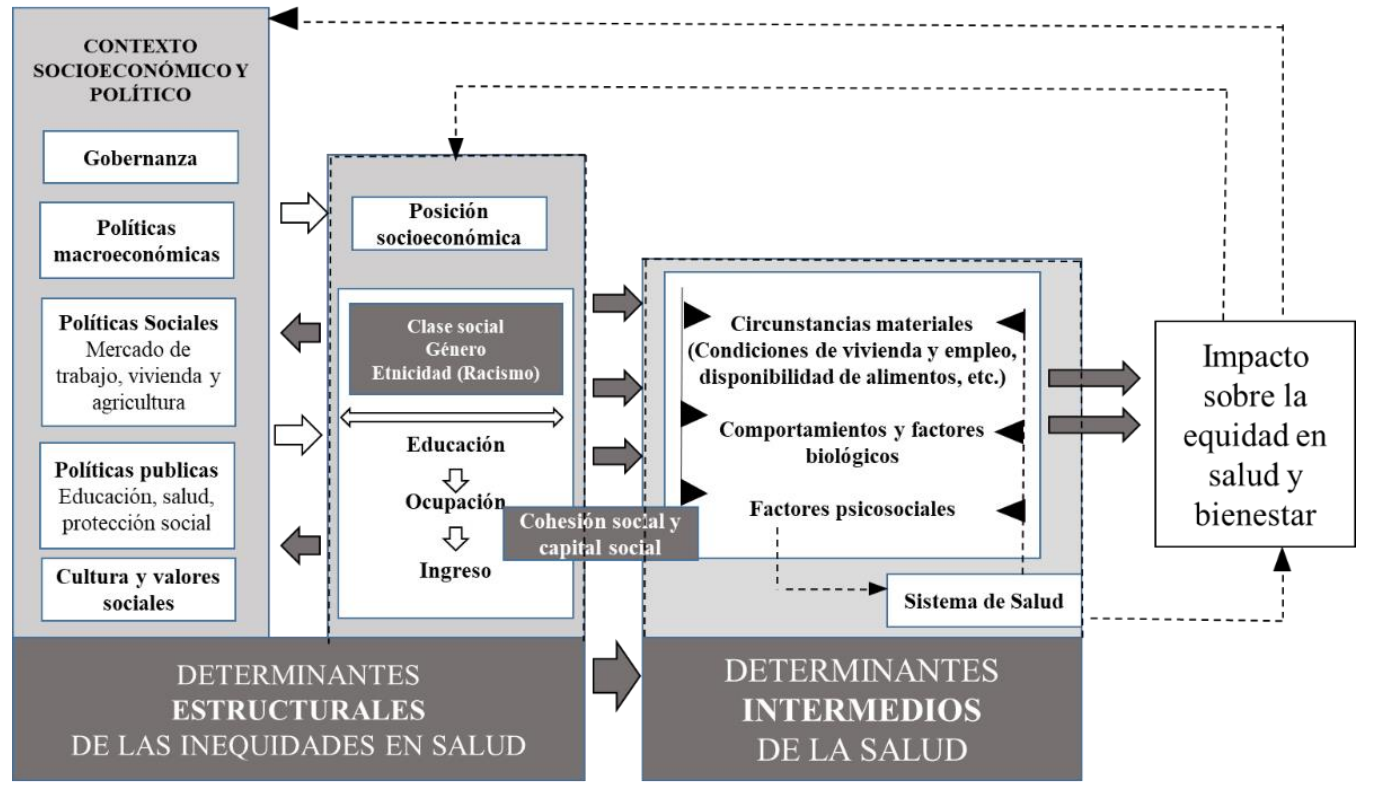

Nota: Solar e Irwin (2010) Comisión de Determinantes Sociales de la OMS. 


\section{Journal of the Academy $|240|$}

\section{Subjetividad y acción}

La subjetividad es un proceso de producción de significados a partir de campos subjetivos y con formas de razonamiento, donde la cultura, vista no como sistema donde la heterogeneidad, discontinuidad y contradicción forman parte de esta, con acumulación social de significados formando configuraciones. Es decir, la producción y la acumulación implican procesos de selección de significados socialmente aceptados, y por niveles de abstracción diversos, en los que las jerarquías de poder de los grupos sociales están presentes, así como la presión de estructuras que si bien están impregnadas de simbolismo no se reducen a lo simbólico, ni los interactuantes tienen por qué estar conscientes de su eficacia para delimitar sus espacios de acción (De la Garza, 2018).

En lo que corresponde a la subjetividad frente a la DT2, en México, algunos estudios sobre creencias de diabetes y tratamiento farmacológico se ha reportado que la enfermedad ha sido atribuida a corajes, sustos, fatalismo: castigo, prueba de dios o atribución al destino (García, Crocker y García de Alba, 2017; Lerin, Juárez y Reartes, 2015). Asimismo, algunas poblaciones originarias de México han considerado al tratamiento farmacológico como dañino para su salud y prefieren usar un tratamiento alternativo, como el uso de hierbas que consideran sanadoras (Ávila, Gómez, Yam, Vega y Franco, 2013; López, Ortiz y Zamora, 2014), lo anterior podría ser resultado de la interacción de los sujetos sociales con las estructuras, que permiten visualizar la enfermedad como una entidad resultado de su propia realidad.

Adicionado a lo anterior, se ha descrito que algunos adultos con DT2 consideran la creencia de que la insulina causa ceguera y temen su uso como tratamiento para la enfermedad. Algunos factores identificados por Vázquez, Lavielle, Gómez y Wacher (2019) sobre el uso de la insulina en pacientes que asisten a unidades de primer nivel de atención fueron: comprensión del paciente frente a la enfermedad y tratamiento, falta de habilidades médicas, miedo a eventos adversos, inseguridad y falta de capacitación. Además, Ávalos, Morfín y Ung (2019) evidenciaron algunos factores socioculturales que intervienen en el rechazo al tratamiento farmacológico como la insulina, al referir que su uso podría quedarlos ciegos; lo cual se relacionó con la escolaridad y el estrato socioeconómico bajos, así como con la falta de información adecuada que el personal de salud brinda, factores que contribuyen al retraso en la insulinización con todas las consecuencias para la salud que esto conlleva. 
Por otra parte, se ha reportado que los adultos con DT2 han manifestado consumir algún remedio no farmacológico para el control de la enfermedad; los remedios más frecuentes para tratar este padecimiento son: Té de hierbas, agua de Jamaica, jugo de cebolla, cacao y jugo de toronja. Algunas otras realidades respecto a tratamientos para la enfermedad fueron: el jugo de limón y de la piña (Salazar et al., 2018). Es por ello por lo que, es necesario considerar las concepciones y significados de los pacientes para lograr mejores resultados, pues, aunque las recomendaciones del personal de salud son muy importantes, no obtendrán resultados de prevención y tratamiento optimo mientras se excluya al paciente como protagonista en decisiones relativas a su salud y enfermedad. (Acosta, García, y Saldaña, 2012; Torres, Sandoval y Pando-Moreno, 2005).

Por su parte López y Ocampo (2007), realizaron un estudio cualitativo para Identificar las creencias sobre su enfermedad, hábitos de alimentación, actividad física y tratamiento de la DT2 en adultos mexicanos, encontraron que los sujetos consideran a la DT2 como un padecimiento que les causa una gran carga emocional y los dirige a un destino inevitable de complicaciones fatales, reconocen como la principal causa al susto o coraje, predomina la idea de una dieta de castigo, consideran los beneficios del ejercicio, sin embargo, no lo realizan, automodifican el tratamiento médico y lo complementan con herbolaria, atribuyen a la insulina como causa de la ceguera. Son conscientes de que su descontrol de la enfermedad se debe a trasgresión en el plan alimentario no obstante persisten en las trasgresiones. Además, detectaron poco interés por el autocuidado.

A continuación, se presenta el esquema de DSS y riesgo de DT2 en población originaria (figura 2). 


\section{Figura 2}

Esquema de los Determinantes Sociales de la Salud y Riesgo de Diabetes Tipo 2 en población originaria, a partir de la estructura, subjetividad y acción.

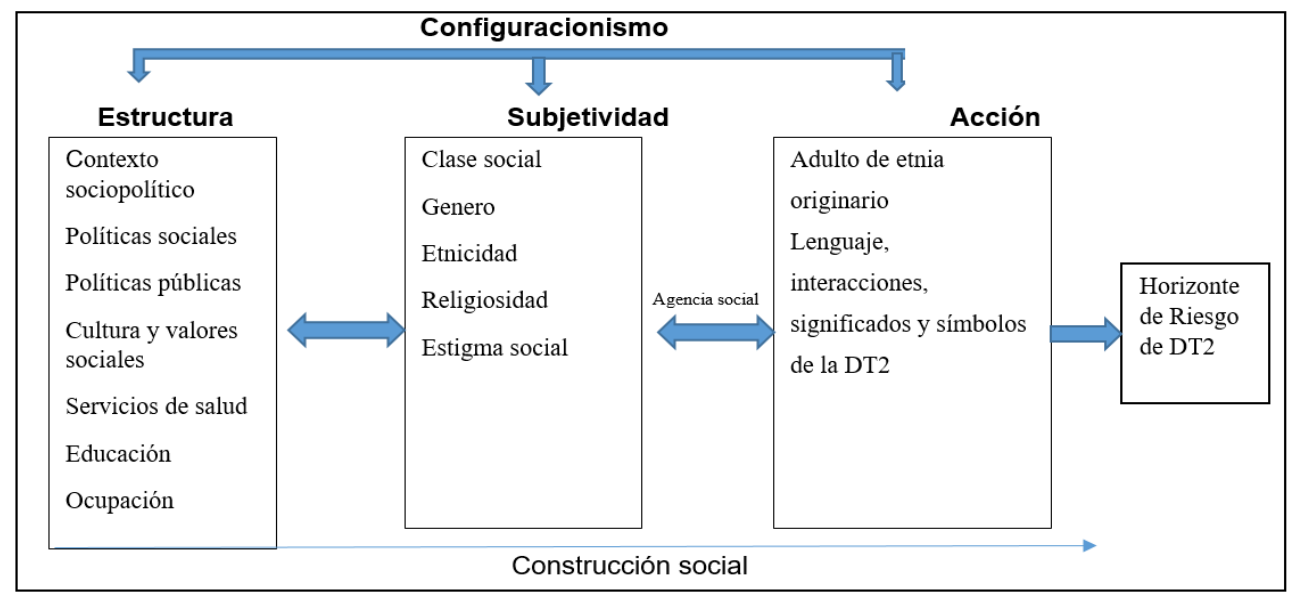

Nota: Elaboración propia

\section{El panorama de la DT2 en la población Originaria Yoreme-mayo}

La población originaria Yoreme-Mayo del norte de Sinaloa se encuentra concentrada en los municipios de El Fuerte, Choix, Guasave, Sinaloa de Leyva y Ahome, debido a su proceso histórico esta población ha compartido su territorio con población mestiza. Como en el resto de México, la globalización ha repercutido en la economía y la salud de la población del estado de Sinaloa, en las últimas décadas, se ha evidenciado un proceso de transición demográfica y epidemiológica, que suman efectos de la modernización y la globalización económica y cultural. Este fenómeno ha impactado en las formas de vida en todo el mundo, especialmente en las poblaciones originarias, quienes han presentado un proceso de cambio en los patrones de alimentación al aumentar en el consumo de alimentos ultraprocesados, cambios en el patrón de actividad física, además de formas de afrontar la salud, enfermedad y atención (Haro, 2007).

Desde la perspectiva de los determinantes de salud, los cambios en patrones de vida y culturización de las comunidades originarias predisponen el aumento del riesgo para padecer 


\section{Journal of the Academy $|243|$}

DT2, esto al vulnerarlos debido a la inequidad, conllevan al casi nulo acceso para cubrir las necesidades básicas como salud, educación, alimento y vivienda, lo cual podría ser resultado de la no inclusión en los planes de desarrollo efectivos en los municipios de estas comunidades, lo que dificulta el logro de los objetivos de la prevención de enfermedades, entre las que destaca la DT2. Las investigaciones en campo y algunos reportes en población Yoreme-Mayo muestran que la necesidad de empleo formal, acceso para la atención a la salud y nutrición, pues hombres y mujeres Yoreme-Mayo en su mayoría desempeñan empleos como jornaleros en campos agrícolas o actividades de empleos en maquiladoras, estás actividades podrían vincularse con problemas de pobreza y privación.

Aunque es difícil precisar el riesgo e impacto de la DT2 de esta población, las estadísticas apuntan a un aumento de la enfermedad en la región norte de Sinaloa, donde se encuentra asentada la población Yoreme-Mayo, sin embargo, las estadísticas siguen imprecisas al no estimar el Sistema de Salud las cifras específicas para esta población. Según datos del Instituto Nacional de los Pueblos Indígenas (2019) la población Yoreme-Mayo del Norte de Sinaloa atiende sus problemas de salud y enfermedad a través de tres vías: por medio de las instituciones oficiales del Sistema Nacional de Salud (medicina alopática), en el hogar (medicina doméstica) y mediante especialistas tradicionales (medicina sociocultural). La medicina doméstica atiende males comunes y se basa en el uso de infusiones como tés y agua de uso con diversas plantas; prácticas con frotamientos y aplicaciones en el cuerpo. Los males más comunes que se curan a través de estos remedios son el dolor de estómago, de muelas, de oído, empacho, fiebre y mollera, entre otras. Sus tratamientos son poco complejos y los realizan principalmente las madres de familia, quienes transmiten los saberes de generación en generación. Sin embargo, estas prácticas se han deteriorado con la institucionalización de la atención de salud, presentando a veces conflictos socioculturales con el uso de medicina alopática, al encontrar renuencia a estos tratamientos.

Por otra parte, encontramos a los especialistas o curanderos que se dedican a atender huesos, espanto, caída de mollera, empacho, partos y se dice que hasta rabia. Sus conocimientos los adquieren por don divino, por enseñanza de algún pariente, mediante un sueño, etcétera. Utilizan rezos, rosarios, oraciones, agua bendita, frotamiento con huevo, sobadas, infusiones, plantas, animales y algunos minerales. En el estado de Sinaloa los curanderos mayos han demandado reconocimiento oficial sobre el ejercicio de su profesión. Sin embargo, esto no ha 


\section{Journal of the Academy $|244|$}

sido posible hasta el momento (INPI, 2019). El panorama de atención de la salud de los Yoreme-Mayo nos permite analizar la importancia de las prácticas tradicionales de atención de la salud para lograr la transversalidad de los cuidados en la prevención de las enfermedades, entre las que destacan la DT2.

Por su parte, las unidades de salud de la Secretaria de Salud de México a través de los Centros de Salud Rurales Dispersos que atiende a población Yoreme-Mayo participa en la prevención de la DT2 a través de la promoción a la salud y prevención de la enfermedad en entornos comunitarios que permiten una interacción muy estrecha con los integrantes de las comunidades, estas unidades de salud son el enlace entre la medicina alopática y tradicional, es aquí es donde se entreteje la interacción sobre el significado de la salud, la enfermedad y la atención de esta población. Es la comunidad el espacio ideal donde dialogan sobre los saberes comunitarios y la medicina que hasta la fecha ha sido en los pueblos originarios una situación pendiente, al persistir el pobre acceso a los servicios de salud, las barreras geográficas y culturales que contribuye a incrementar la marginación y aumentar las desigualdades en salud (Menéndez, 2008).

En la población Yoreme-Mayo, poco se ha estudiado respecto a temas de salud, Sin embargo, algunas características sociodemográficas son similares a otros grupos indígenas de México que podrían ser motivo de interés para estudios a profundidad, la religión es una categoría muy importante, se estima que más del $90 \%$ de la población practica alguna religión, siendo la religión católica la de mayor práctica en poblaciones originaria y cuyos rituales se basan en cultos religiosas afines al ritual católico, dichas prácticas se organizan en torno a los centros ceremoniales o pueblos tradicionales, formados por comunidades más pequeñas congregadas en adoración y rito a un santo. En cada uno de ellos se desempeñan una serie de cargos designados por las autoridades religiosas, como el maestro rezador, las cantoras y la directiva de la iglesia (INPI, 2019).

En lo que corresponde al peso corporal, el Sobrepeso y Obesidad un problema en población indígena y no indígena, siendo más frecuente en mujeres, esto podría deberse a cambios en el patrón de alimentación y transición de una alimentación tradicional a incluir en su dieta alimentos ultraprocesados de bajo costo. Conviene analizar que la población Yoreme-Mayo no dispone actualmente de datos sobre la situación de salud, sin embargo, presenta características 


\section{Journal of the Academy $\mid 245$ |}

sociodemográficas similares a reportes de estudios en Mixtecos, Pimas, Yaquis y Wixaricas al tener nivel de escolaridad bajo (primaria) e ingreso económico por debajo de la media de salario mínimo, esta situación aumenta la inequidad al no cubrir las necesidades de alimentación, seguridad social y acceso a una vivienda digna (Consejo Nacional de Evaluación de la Política de Desarrollo Social [CONEVAL], 2018; García, Crocker y Alba, 2017; Jiménez et al., 2019; Rodríguez et al., 2008), además de vulnerarlos frente a falta de protección y desventajas que enfrentan para resolver los problemas de salud en comparación con otros grupos de población (Juárez et al, 2014).

En cuanto a la ocupación, resalta que los Yoreme-Mayo, sobre todo los hombres realizan trabajos de jornaleros que se caracterizan por cumplir con actividades que requieren de actividad física vigorosa. Esparza et al., (2015) mostraron que realizar actividad física de moderada a vigorosa en trabajos de cultivos es un factor protector para DT2 en indígenas Pimas de Sonora. En el caso de las mujeres, aunque ha aumentado la actividad laboral, siguen realizando actividades del hogar, lo que sugiere que la mujer Yoreme-Mayo sigue desarrollando un papel tradicional al realizar actividades para el mantenimiento del hogar y crianza de los hijos, estas características sociodemográficas posicionan a este grupo originario en una situación de inequidad y pobreza (INPI, 2019; Goodman, Fraga, Brodine, Ibarra y Garfein, 2013; Loria, Arroyo, Fernández, Pardio y Laviada, 2018). Los datos anteriores dan una pincelada de la situación actual de la DT2 en población Yoreme-Mayo, sin embargo, es necesario abordar el fenómeno a profundidad desde la estructura, subjetividad y acción, que permita comprender el horizonte de riesgo para DT2 de la población Yoreme-Mayo.

\section{CONCLUSIONES}

Es necesario considerar las estructuras, subjetividad y acción como un marco para el estudio sobre los resultados de la salud de las poblaciones originarias, a pesar de la importancia del abordaje de la salud-enfermedad desde la perspectiva social, pocos estudios sobre riesgo de DT2 se han abordado desde este sendero. La comprensión del lenguaje, símbolos, valores, interacciones y significados podría acercarnos a ofrecer mejores estrategias que permeen en la disminución del riesgo de enfermar en los diversos grupos poblacionales, sobre todo en grupos originarios, que por condiciones de inequidad y vulnerabilidad los ponen en desventaja frente a la enfermedad y por ende a un curso de vida menos virtuoso. 


\section{Journal of the Academy $\mid 246$ |}

A partir de lo expuesto, es necesario realizar estudios sociales de la experiencia de vida tratando de encontrar las conexiones entre las estructuras y las formas en cómo estas se posicionan sobre el cuidado de la salud, a ello se suman la multiplicidad de significados que cada una de las personas forma de la salud, su cuidado y el afrontamiento de la enfermedad.

Se debe de dirigir los esfuerzos para brindar una atención y compresión hacia sus creencias y prácticas, formas de afrontar los riesgos para la enfermedad; considerarlas en el plan de cuidado integral, asimismo los profesionales de salud que cuidan a pacientes con esta enfermedad deben de conocer el contexto cultural de sus pacientes, de esta forma podríamos acercarnos a un tratamiento con resultados efectivos.

En este sentido llaman la atención sobre dos aspectos fundamentales: la necesidad de articular las miradas emic/etic en los diferentes análisis que se hagan de los datos que sirvan para la comprensión de los fenómenos sociales y la salud-enfermedad, y la importancia de claramente distinguir la subjetividad que orienta las prácticas hacia el cuidado de la salud y las formas de afrontar la enfermedad, sobre todo cuando se refiere a representaciones de las prácticas, que frecuentemente son confundidas con la práctica misma. Lo expuesto en trabajo podría servir de apoyo para direccionar la comprensión de los horizontes de riesgo a la salud y enfermedad en pueblos vulnerables.

\section{REFERENCIAS BIBLIOGRÁFICAS}

Acosta, L., García, A., \& Saldaña, K. (2012). Concepciones culturales sobre insulinoterapia de pacientes diabéticos tipo 2. RESPYN Revista Salud Pública y Nutrición, 13(1). Recuperado de http://respyn.uanl.mx/index.php/respyn/article/view/301

Asociación Americana de Diabetes. (2019). Introduction: Standards of Medical Care in Diabetes-2019. Diabetes Care, 42(1), 1-2. https://doi.org/10.2337/dc19-S002

Asociación Americana de Diabetes. (2020). Standards of Medical Care in Diabetes-2020. Diabetes Care, 43(1), 7-13.

Asociación Latinoamericana de Diabetes. (2019). Guías ALAD sobre el Diagnóstico, Control y Tratamiento de la Diabetes Mellitus Tipo 2 con Medicina Basada en Evidencia Edición 2019.

Recuperado

de https://www.revistaalad.com/guias/5600AX191_guias_alad_2019.pdf 
Ávalos, A.H., Morfín, M.C.J., y Ung, M.E. (2019). Factores socioculturales que intervienen en el rechazo al uso de insulina. Atención Familiar, 26(2), 58-62. https://www.medigraphic.com/cgi-bin/new/resumen.cgi?IDARTICULO=86046

Ávila, G. M., Gómez, P., Yam, A. V., Vega, G., \& Franco, B. E. (2013). A Qualitative Approach to Barriers to Adherence to Therapy among the Chronically Ill in Guanajuato, Mexico. Aquichan, 13(3), 373-386

Banco Mundial. (2018). Pueblos indígenas: Panorama general. Recuperado de https://www.bancomundial.org/es/topic/indigenouspeoples

Bari, A., y Cristina, M. (2002). La cuestión étnica: Aproximación a los conceptos de grupo étnico, identidad étnica, etnicidad y relaciones interétnicas. Cuadernos de Antropología Social, 16(1), 149-163. Recuperado de http://www.redalyc.org/articulo.oa?id=180913908002

Basilio, C. D., Kwan, V. S., y Towers, M. J. (2016). Culture and risk assessments: Why Latino Americans perceive greater risk for diabetes. Cultural Diversity and Ethnic Minority Psychology, 22(1), 104-113. https://doi.org/10.1037/cdp0000034

Benach, J., y Amable, M. (2004). Las clases sociales y la pobreza. Gaceta Sanitaria, 18(4), 1623. Recuperado de http://scielo.isciii.es/scielo.php?script=sci_arttext\&pid=S021391112004000400005\&lng=es\&tlng=es

Bourdieu, P. (2017). Habitus. In Habitus: A sense of place. Routledge pp. 59-66.

Breilh, J. (2013). La determinación social de la salud como herramienta de transformación hacia una nueva salud pública (salud colectiva). Revista Facultad Nacional de Salud Pública, $31(1)$, $13-27$. http://www.scielo.org.co/scielo.php?script=sci_arttext\&pid=S0120$\underline{386 \times 2013000400002}$

Caballero, A. E. (2018). The "A to Z" of managing type 2 diabetes in culturally diverse $\begin{array}{lllll}\text { populations. } \quad \text { Frontiers in } & \text { Endocrinology, } & \text { 9, }\end{array}$ https://doi.org/10.3389/fendo.2018.00479

Cardona, D., Acosta, L. D., y Bertone, C. L. (2013). Inequidades en salud entre países de Latinoamérica y el Caribe (2005-2010). Gaceta Sanitaria, 27(4), 292-297.

Castro, A., Serna, A., Lozoya, J., Toledo, I., Día, R., y Esparza, J. (2018). Prevalence of previous diagnosis of hypertension and associated factors in the Yaqui indigenous of Sonora. Revista Mexicana de Cardiología, 29(2), 90-97. Recuperado de https://www.medigraphic.com/cgibin/new/resumenI.cgi?IDARTICULO=80464

Consejo Nacional de Evaluación de la Política de Desarrollo Social, CONEVAL. (2018). Pobreza en México: Resultados de pobreza en México 2018 a nivel nacional y por entidades federativas. Recuperado de https://www.coneval.org.mx/Medicion/MP/Paginas/Pobreza-2018.aspx 
De la Garza, E. (2001). Subjetividad, cultura y estructura. Iztapalapa. Revista de Ciencias Sociales $y$ Humanidades, 5, 83-101. Recuperado de https://revistaiztapalapa.izt.uam.mx/index.php/izt/article/view/527/680

De la Garza, E. (2018). La metodología configuracionista para la investigación social. En (E. De la Garza-Toledo (ed.); Primera Edición, Vol. 1). Editorial Gedisa, SA. Recuperado de http://www2.izt.uam.mx/sotraem/NovedadesEditoriales/MetodologiaConfig_SD.pdf

De la Torre-Ugarte-Guanilo, M., \& Oyola-García, A. (2014). Los determinantes sociales de la salud: una propuesta de variables y marcadores/indicadores para su medición. Revista Peruana de Epidemiología, 18(1), 1-6. Recuperado de https://www.redalyc.org/articulo.oa?id=2031/203132677002

Egeland, G. M., Cao, Z., y Young, T. K. (2011). Hypertriglyceridemic-waist phenotype and glucose intolerance among Canadian Inuit: the International Polar Year Inuit Health Survey for Adults 2007-2008. Canadian Medical Association Journal, 183(9), 553-558. https://doi.org/10.1503/cmaj.101801

Encuesta Nacional de Salud y Nutrición (2018). Últimas cifras de diabetes en México. Recuperado de https://ensanut.insp.mx/encuestas/ensanut2018/index.php

Encuesta Nacional de Salud y Nutrición. (2012). Encuesta Nacional de Salud y Nutrición 2012, Resultados. https://www.insp.mx/images/stories/ENSANUT/norte/Sinaloa-NOR.pdf

Encuesta Nacional de Salud y Nutrición. (2016). Informe de resultados. Recuperado de https://ensanut.insp.mx/encuestas/ensanut2016/index.php

Engel, G. (1977). La necesidad de un nuevo modelo médico: un reto para la biomedicina. Revista ciencia, 196(4282), 129, 136.

Esparza, J., Valencia, M. E., Urquidez, R., Chaudhari, L. S., Hanson, R. L., Knowler, W. C.,... Schulz, L. O. (2015). Environmentally Driven Increases in Type 2 Diabetes and Obesity in Pima Indians and NonPimas in Mexico Over a 15-Year Period: The Maycoba Project. Diabetes Care, 38(11), 2075-2082. https://doi.org/10.2337/dc15-0089

García-Serrano, V., Crocker, R. y García de Alba, J. (2017). Creencias sobre la diabetes mellitus tipo 2 de la etnia Wixarika del occidente de México. Revista Chilena de Antropología, 35, 113-130. https://doi.org/10.5354/0719-1472.2017.46151

Giddens, A. (1998) La Construcción de la Sociedad. Buenos Aires: Amorrortu

Goodman, D., Fraga, M. A., Brodine, S., Ibarra, M.-L., y Garfein, R. S. (2013). Prevalence of Diabetes and Metabolic Syndrome in a Migrant Mixtec Population, Baja California, Mexico. Journal of Immigrant and Minority Health, 15(1), 93-100. https://doi.org/10.1007/s10903-012-9717-0

Gutiérrez, J., Heredia-Pi, I., Hernández-Serrato, M., Pelcastre-Villafuerte, B., Torres-Pereda, P., y 
Reyes-Morales, H. (2019). Desigualdades en el acceso a servicios, base de las políticas para la reducción de la brecha en salud. Salud Pública de México, 61(6), 726-733. https://doi.org/10.21149/10561

Haro-Encinas, J. A. (2007). Globalización y salud de los trabajadores: Jornaleros agrícolas y producción de uva en Pesqueira, Sonora. Región y sociedad, 19(40), 73-105. Recuperado de $\quad$ http://www.scielo.org.mx/scielo.php?script=sci_arttext\&pid=S187039252007000300003\&lng=es\&tlng=es

Instituto Nacional de Diabetes y Enfermedades Digestivas y Renales, NIDDK. (2020). Factores de riesgo de diabetes. Recuperado de https://www.niddk.nih.gov/healthinformation/informacion-de-la-salud/diabetes/informacion-general/que-es

Instituto Nacional de los Pueblos Indígenas. (2019). Pueblos Indígenas - Atlas de los Pueblos Indígenas de México. Recuperado de http://atlas.cdi.gob.mx/?page id=67

International Diabetes Federation. (2019). IDF Diabetes Atlas 9th edition. Recuperado de https://www.diabetesatlas.org/en/

Iriart, C., Waitzkin, H., Breilh, J., Estrada, A., y Merhy, E. E. (2002). Medicina social latinoamericana: aportes y desafíos. Revista Panamericana de Salud Pública, 12, 128136

Jiménez, A., Nelson, R. G., Jimenez, M. E., Franks, P. W., Aguilar, C. A., Graue, E. O., Hernandez, J., y Hernández, M. (2019). Disparities in prediabetes and type 2 diabetes prevalence between indigenous and nonindigenous populations from Southeastern Mexico: The Comitan Study. Journal of Clinical \& Translational Endocrinology, 16. https://doi.org/10.1016/J.JCTE.2019.100191

Juárez, C., Márquez, M., Salgado, N., Pelcastre, B. E., Ruelas, M. G., y Reyes, H. (2014). Health inequality among vulnerable groups in Mexico: older adults, indigenous people, and migrants. Revista Panamericana de Salud Pública, 35, 284-290.

Juárez-Ramírez, C., Théodore, F., Villalobos, A., Allen-Leigh, B., Jiménez-Corona, A., Nigenda, G. y Lewis, S. (2019). La importancia de la dimensión cultural de la comida para comprender la falta de adherencia a los regímenes dietéticos entre los mayas con diabetes. Nutrición de salud pública, 22 (17), 3238-3249. doi:10.1017 / S1368980019001940

Leyva, R., Infante, C., Gutierrez, J. P., y Quintino, F. (2013). Inequidad persistente en salud y acceso a los servicios para los pueblos indígenas de México, 2006-2012. Salud Pública de México, 55, 123-128

Lerin P. S., Juárez, C. \& Reartes. (2015). Creencias de indígenas chiapanecos en torno a la diabetes y posibilidades de atención intercultural. Salud Problema, (17), 27-41

Linares, N., y López, O. (2003). La equidad en salud: propuestas conceptuales, aspectos críticos y perspectivas desde el campo de la Salud Colectiva. Medicina Social, 3(3), 247259. https://socialmedicine.info/index.php/medicinasocial/article/view/226 
López, K., y Ocampo, P. (2007). Creencias sobre su enfermedad, hábitos de alimentación, actividad física y tratamiento en un grupo de diabéticos mexicanos. Archivos En Medicina Familiar, 9(2), 80-86. Recuperado de http://www.redalyc.org/articulo.oa?id=50711454003

López, L. E., Ortiz, A., \& Zamora, K. (2014). Mecanismos multiculturales de indígenas residentes de la ciudad de Pachuca Hidalgo. Revista Mundo Siglo XXI, 34(10), 75-83

Loria, A., Arroyo, P., Fernandez, V., Pardio, J., y Laviada, H. (2018). Prevalence of obesity and diabetes in the socioeconomic transition of rural Mayas of Yucatan from 1962 to 2000. Ethnicity \& Health, 1-7. https://doi.org/10.1080/13557858.2018.1442560

Meljem-Moctezuma, J. (2016). Conformación del Observatorio de Inequidades en Salud en México. Salud Pública de México, 58(6), 606-607. https://doi.org/10.21149/spm.v58i6.8397

Menéndez, E. L. (2016). Salud intercultural: propuestas, acciones y fracasos. Ciência \& Saúde Coletiva, 21, 109-118

Menéndez, E. L. (2008). Epidemiología sociocultural: propuestas y posibilidades. Región y sociedad, 20(2), 5-50. $\quad$ Recuperado de http://www.scielo.org.mx/scielo.php?script=sci_arttext\&pid=S1870$\underline{39252008000400002 \& \operatorname{lng}=\mathrm{es} \& \ln g=}$

Mundo-Rosas, V., Unar-Munguía, M., Hernández-F, M., Pérez-Escamilla, R., y Shamah-Levy, T. (2019). La seguridad alimentaria en los hogares en pobreza de México: una mirada desde el acceso, la disponibilidad y el consumo. Salud Pública de México, 61(6), 866875. https://doi.org/10.21149/10579

Niessen, L. W., Mohan, D., Akuoku, J. K., Mirelman, A. J., Ahmed, S., Koehlmoos, T. P.,... Peters, D. H. (2018). Tackling socioeconomic inequalitiesí and non-communicable diseases in low-income and middle-income countries under the Sustainable Development agenda. The Lancet, 391(10134), 2036-2046. https://doi.org/10.1016/s0140$\underline{6736(18) 30482-3}$

Organización Mundial de la Salud. (2008). Comisión sobre Determinantes sociales de la salud - Informe $\quad$ Final. $\quad$ Recuperado de https://www.who.int/social determinants/thecommission/finalreport/es/

Organización Mundial de la Salud. (2009). Determinantes sociales de la Salud. Recuperado de https://apps.who.int/gb/ebwha/pdf_files/A62/A62_R14-sp.pdf?ua=1

Organización Mundial de la Salud. (2016). Informe mundial sobre la diabetes 2016. WHO; World Health Organization. Recuperado de https://www.who.int/diabetes/globalreport/es/

Organización Mundial de la Salud. (2018). Diabetes. Recuperado de http://www.who.int/mediacentre/factsheets/fs312/en/ 
Organización Mundial de la Salud. (2020). Determinantes sociales de la salud. Recuperado de https://www.who.int/social_determinants/es/

Pacheco, L. S., Hernández-Ontiveros, D. A., Iniguez-Stevens, E., Brodine, S., Garfein, R. S., Santibañez, M., y Fraga, M. A. (2018). Prevalence and correlates of diabetes and metabolic syndrome in a rural indigenous community in Baja California, Mexico. BMC Public Health, 18(1), 1397. https://doi.org/10.1186/s12889-018-6276-X

Rodríguez, M., Guerrero, F., Brito, O., Rascón, R. A., Pérez, R., Sánchez, M. C.,... Sánchez, J. (2008). Cardiovascular Risk Factors and Acculturation in Yaquis and Tepehuanos Indians from Mexico. Archives of Medical Research, 39(3), 352-357. https://doi.org/10.1016/j.arcmed.2007.12.003

Salazar, F.E., Ponce, R.E., Jiménez. G.I., Cervantes, N. A., Jiménez-Hernández, J. C., y Madrigal-de León, H. G. (2018). Mitos y creencias sobre la diabetes en pacientes de una unidad médica de atención primaria en la Ciudad de México. Archivos de Medicina Familiar, 20(1), 5-21. Recuperado de https://www.medigraphic.com/cgibin/new/resumen.cgi?IDARTICULO $=76224$

Si, D., Bailie, R., Wang, Z., y Weeramanthri, T. (2010). Comparison of diabetes management in five countries for general and indigenous populations: an internet-based review. BMC Health Services Research, 10(1). https://doi.org/10.1186/1472-6963-10-169

Solar, O., y Irwin, A. (2010). A conceptual framework for action on the social determinants of health. Social Determinants of Health Discussion Paper 2 (Policy and Practice). Recuperado de https://apps.who.int/iris/handle/10665/44489

Torres-López, T. M., Sandoval-Díaz, M., y Pando-Moreno, M. (2005). "Sangre y azúcar": representaciones sobre la diabetes de los enfermos crónicos en un barrio de Guadalajara, México. Cadernos de Saúde Pública, 21(1), 101-110. https://doi.org/10.1590/S0102$\underline{311 X 2005000100012}$

Vázquez, F., Lavielle, P., Gómez-Díaz, R., \& Wache, N. (2019). Inercia clínica en el tratamiento con insulina en el primer nivel de atención. Gaceta Medica de México, 155(2), 156-161. Recuperado de https://www.medigraphic.com/cgibin/new/resumen.cgi?IDARTICULO $=86523$

Villalobos, A., Rojas-Martínez, R., Aguilar-Salinas, C. A., Romero-Martínez, M., MendozaAlvarado, L. R., Flores-Luna, M. de L., Escamilla, A., y Ávila-Burgos, L. (2019). Atención médica y acciones de autocuidado en personas que viven con diabetes, según nivel socioeconómico. Salud Pública De México, 61(6), 876-887. https://doi.org/10.21149/10546 